\title{
A COMPARATIVE STUDY ON DRUG USE OF ANTIMICROBIAL AGENTS IN MEDICAL AND SURGICAL INTENSIVE CARE UNITS OF A TERTIARY CARE HOSPITAL
}

\author{
NIMIN PAUL ${ }^{1 *}$, SANTHOSH KUMAR V \\ ${ }^{1}$ Department of Pharmacy Practice, School of Pharmaceutical Sciences, Vistas, Vels University, Pallavaram, Chennai, Tamil Nadu, India. \\ ${ }^{2}$ Department of Pharmacology, School of Pharmaceutical Sciences, Vistas, Vels University, Pallavaram, Chennai, Tamil Nadu, India. \\ Email: niminpaulzz@gmail.com
}

Received: 26 August 2017, Revised and Accepted: 21 October 2017

ABSTRACT

Objective: To identify the pattern of drug utilization of antimicrobials in prescriptions of patients admitted at medical intensive care unit (MICU) and surgical intensive care unit (SICU) department and to analyze the utilization of different classes of drugs.

Methods: A prospective observational study was carried out in 10 bedded medical and surgical intensive care unit (ICU) of tertiary care hospital, Adayar, from October $1^{\text {st }} 2016$ to March $1^{\text {st }} 2016$. The study was performed in 100 prescriptions. The relevant data on drug prescription of each patient were collected from in-patient records. The demographic data, disease data, and the utilization of different classes of antimicrobial agents (AMAs) were analyzed.

Results: In MICU, a total of 648 drugs were prescribed during the period of stay and AMAs accounted for $15 \%$ of total drug. The average length of stay was found to be $8( \pm 8.73)$. The mean number of drugs received by patients is $11.6 \pm 2$ drugs. The most frequently prescribed AMAs were ceftriaxone followed by meropenem and clindamycin. Cephalosporin is commonly prescribed due to their relatively lower toxicity and broader spectrum activity. The generally prescribed AMA combination was amoxicillin + clavulanic acid (32.50\%) and piperacillin + tazobactam (27.50\%). The laboratory reported positive cultures for 30 patients. The most prevailing organisms were Escherichia coli (50\%). In SICU, a total of 780 drugs were prescribed during the period of stay and AMAs accounted for $18 \%$ of total drug. An average of $5( \pm 2.0)$ drugs was prescribed for each patient and each prescription contains an average of $2( \pm 0.9)$ AMAs. The results indicated that ceftriaxone was the most commonly prescribed AMAs $(22 \%)$, followed by meropenem (18\%), ciprofloxacin (18\%), and colistin (8\%). A total of five AMA combination therapies were used in SICU. Among them, piperacillin+clavulanic acid (36.84\%) was the most commonly prescribed combination. In the study, 30 (60 \%) cases had microbial growth and have performed sensitivity test.

Conclusion: A wide class and percentage of AMAs were prescribed in ICUs. There is a need of antimicrobial agent's usage guidelines and restriction policies for the rational prescribing of antimicrobials in critically ill patients.

Keywords: Drug utilization study, Antimicrobial agents, Medical intensive care unit, Surgical intensive care unit.

(C) 2018 The Authors. Published by Innovare Academic Sciences Pvt Ltd. This is an open access article under the CC BY license (http://creativecommons. org/licenses/by/4. 0/) DOI: http://dx.doi.org/10.22159/ajpcr.2018.v11i1.22259

\section{INTRODUCTION}

Drug therapy plays a major role in enhancing the quality of life and extending the life expectancy of human health. A method to evaluate and improve drug use is by conducting drug utilization studies (DUS) [1]. Drug utilization review was defined by the WHO in 1977 as the marketing, distribution, prescription, and use of drugs in a society, with special emphasis on the resulting medical, social, and economic consequences [2].

The principal aim of DUS is to facilitate the rational use of drugs in populations. For the individual patient, the rational use of a drug means patients receive medications appropriate to their clinical needs. Without knowledge of how drugs are being prescribed, it is difficult to suggest measures to improve prescribing habits. Pharmacists play a key role in this process because of their expertise in the area of pharmaceutical care.

Intensive care unit (ICU) patients are seriously ill patients require close observation and monitoring who often suffer from multiple organ dysfunctions. These patients have high mortality and morbidity and require a high level of intensive care [3]. Selection of drugs and initiating a drug therapy in critical care patients is a complex task and is usually difficult.
Antimicrobials are agents that destroy microorganisms capable of causing diseases. Antimicrobials are used and misused widely since their discovery for treatment and prevention of various disease conditions [4]. They are the commonly prescribed drugs in an ICU setting. In such circumstances, optimal antibiotic use is crucial in the critical care setting, especially in an era of rising antibiotic resistance and lack of new antimicrobial development [5]. To improve antimicrobial use, its outcome needs to be measured by introducing a proper guideline and monitoring process for the use of antimicrobial agents (AMAs). Such practices are highly beneficial as it can ensure rational drug therapy.

DUS are important for obtaining data about the patterns and quality of use, the determinants of drug use, and the outcomes of use [6]. It serves as a means of improving the quality of patient care and enhancing therapeutic outcomes, which help to set priorities for the rational allocation of health-care budgets.

The objective of this study is to identify the pattern of drug utilization of antimicrobials in prescriptions of patients admitted at medical ICU (MICU) and surgical ICU (SICU) department and to analyze the utilization of different classes of drugs. 


\section{METHODS}

A prospective observational study was carried out for 6 months. The study was conducted in MICU and SICU of 180 bedded hospital. A total of 100 prescriptions were included in the study (MICU-50, SICU 50).The inclusion criteria were patients above 15 years and who were admitted in MICU and SICU. Patients with incomplete data and treatment charts without AMAs were excluded from the study. Permission to collect data was taken from the head of surgical ICU before starting the study. The data sources for the study were collected from case reports, treatment charts, and laboratory reports in a specially designed pro forma containing relevant details such as demographics (age, sex, and outcomes of the patient), clinical data (clinical diagnosis, length of ICU stay), and drug data. Drugs prescribed (generic/brand name) dosage route frequency of administration were collected as per pro forma.

\section{Statistical analysis}

Data were entered into Microsoft Excel (Windows 10; version 2013) and subjected to descriptive analysis. Mean and standard deviation (SD) was computed from continuous variables. Graphic representation was used for visual interpretation of the analyzed data.

\section{Ethics}

The study protocol was assessed and approved by the institutional ethics committee of Vels University. Confidentiality of data was maintained.

\section{RESULTS AND DISCUSSIONS}

Antibiotics are one of the most common pharmacotherapies administered in the ICU setting [7]. The clinical setting in the ICU warrants the use of drugs from various drug classes. The crucial step in any intervention program to improve drug use is to assess the existing problem in prescribing [8]. DUE is an inexpensive, flexible, and simple method to assess the utilization pattern of drug [9]. The present study was performed to evaluate and improve the use of antimicrobials in critical care patients. The objective of our study was to identify the pattern of drug utilization of antimicrobials in prescriptions of patients admitted at MICU and SICU department and to analyze the utilization of different classes of drugs.

During the 6 months study period, a total of 100 prescriptions were analyzed from various ICU (MICU-50, SICU-50). In MICU, the highest rate of drug prescription was observed for patients aged between 46 and 60 years, which consist of $15(30 \%)$ female and $35(70 \%)$ male. Mean \pm SD for age was found to be $25 \pm 3.405877$. In SICU, the highest rate of drug prescription was observed for patients aged between 61 and 75 years, which consist of $52 \%$ males. Mean \pm SD for age was found to be $25 \pm 4.560702$ (Table 1). MICU is a unit providing acute care for adult and geriatric critically ill patients. The demographic results of patients admitted to the MICU revealed male preponderance with a mean age of $50( \pm 14.14)$ years and the average length of stay (LOS) was found to be $8( \pm 8.73$ ) days (Fig. 1). The data were similar to studies conducted by Shankar et al. in ICU of a teaching hospital in Western Nepal [10]. Previous Indian study also documented male predominance which suggests that more males are admitted to the Indian setting [11].

\section{Admitting diagnosis of the study population}

A wide spectrum of clinical diagnoses was observed. Chest infection was the most common comorbidity. Among the 100 admitted patients, 27 shows chest infection. The other most common admitting diagnosis were hernia (15\%), chronic obstructive pulmonary disease acute exacerbation (13\%), status asthmaticus (11\%), pulmonary edema (8\%), pneumonia (7\%), and pleural effusion (6\%) (Fig. 2). singh $\mathrm{N}$ et al . had reported that ICU contributes $20-30 \%$ of the nosocomial infections in the hospital [11]. It was observed that $70.5 \%$ patients admitted to the medical ICU had more than three comorbid conditions with hypertension (32.5\%), diabetes mellitus (23.6\%), and ischemic heart disease $(11.5 \%)$ as the major comorbid conditions. "Comorbidity" increases the total burden of the illness in a patient and also contributes to clinical outcomes as well as to economic outcomes [12].

\section{AMAs prescribed in study population}

In MICU, a total of 648 drugs were prescribed during the period of stay and AMAs accounted for $15 \%$ of total drug. In SICU, a total of 780 drugs were prescribed during the period of stay and AMAs accounted for $18 \%$ of total drug. In MICU, the mean number of drugs received by patients (11.6 \pm 2 drugs) was comparable to Smythe et al. study $(12 \pm 7.6$ drugs) [13] but higher compared to report from Nepal in 2005 which recorded a mean of 5.1 \pm 2.7 drugs [14]. Similarly, studies from Danish University ICU hospital shows many of their patients were prescribed with only one AMA [15]. In our observation, most of the patients received one or two AMAs.

Table 1: Demographic profile of study population

\begin{tabular}{|c|c|c|}
\hline \multirow[t]{2}{*}{ Gender } & \multicolumn{2}{|l|}{$n=50(\%)$} \\
\hline & MICU & SICU \\
\hline Male & 35 (70.00) & $26(52.00)$ \\
\hline Female & $\begin{array}{l}15(30.00) \\
25 \pm 10\end{array}$ & $\begin{array}{l}24(48.00) \\
25 \pm 1\end{array}$ \\
\hline \multicolumn{3}{|c|}{ Age distribution } \\
\hline $15-30$ & $5(10.00)$ & $3(6.00)$ \\
\hline $31-45$ & $8(16.00)$ & $7(14.00)$ \\
\hline $46-60$ & $15(30.00)$ & $13(26.00)$ \\
\hline $61-75$ & $12(24.00)$ & $16(32.00)$ \\
\hline \multirow[t]{2}{*}{$>75$} & $10(20.00)$ & $11(22.00)$ \\
\hline & $25 \pm 3.405877$ & $25 \pm 4.560702$ \\
\hline
\end{tabular}

MICU: Medical intensive care unit, SICU: Surgical intensive care unit, SD: Standard deviation

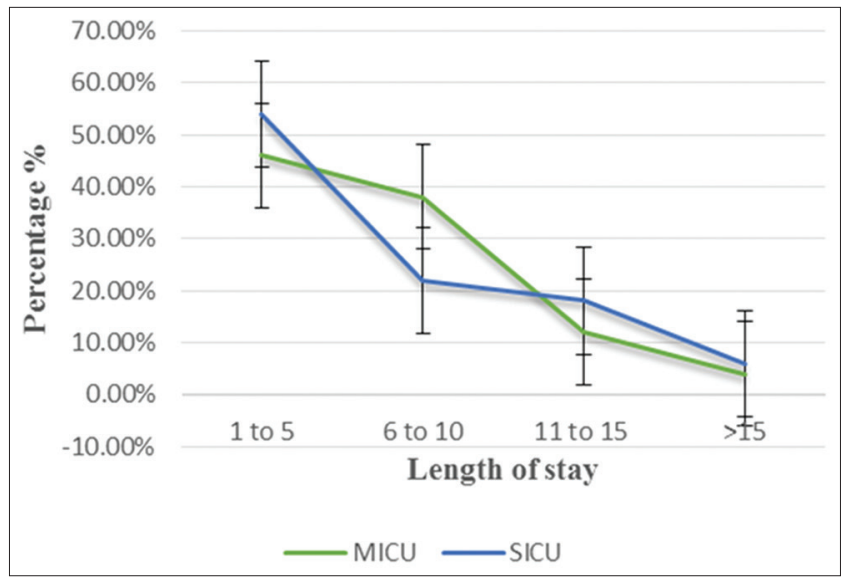

Fig. 1: Length of stay of the study population

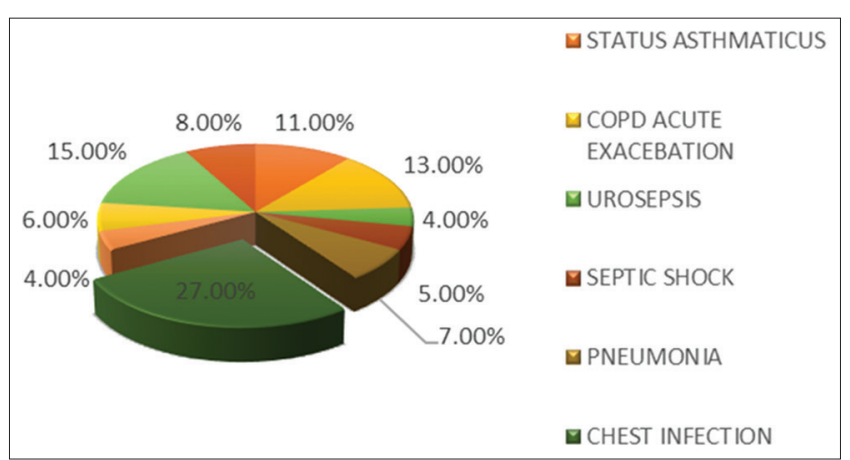

Fig. 2: Admitting diagnosis of the study population 
Table 2 summarizes the most frequently prescribed AMAs in MICU and SICU. In MICU, ceftriaxone usage was found to be more (25\%) as compared to other AMAs followed by meropenem, clindamycin, cefotaxime, colistin, and ciprofloxacin. In SICU, the most frequently prescribed AMAs is ceftriaxone followed ciprofloxacin, clindamycin, and erythromycin. The AMAs in MICU were found to vary in comparison to other study in India (data not reported). Higher utilization of cephalosporins (60\%) and aminoglycosides (34\%) noticed, similar to Usluer et al. study [16] but differed from Shankar et al. in which penicillins were the most common antimicrobial drug class prescribed. Cephalosporins are commonly prescribed due to their relatively lower toxicity and broader spectrum activity.

\section{AMAs prescribed in combination}

The most frequently prescribed AMAs combination in MICU and SICU are summarized in Table 3. It was observed that amoxicillin + clavulanic acid combination (32.50\%) was found to be prescribed more in MICU, followed by piperacillin+tazobactam (27.50\%). In SICU, the most frequently prescribed AMA combination is amoxicillin+clavulanic acid (36.84\%), followed by piperacillin+tazobactam (28.95\%).

\section{Pattern of culture in MICU and SICU}

The laboratory reported positive cultures for 30 patients. The most prevailing organisms were Escherichia coli (53\%) and Acinetobacter sps. (23\%). Colistin and meropenem were the most sensitive AMA for most microorganisms. Most growth of the microbial was found in blood (13 no's) followed by urine and sputum. No growths were found in pleural fluid (Figs 3 and 4).

The prescription of AMAs in the hospital setting is often empiric, especially in the critically ill patients. In our study site, the total number of beds in MICU and SICU was 10 each and the occupancy indices of MICU and RICU were 0.7 and 0.6 , respectively. The demographic results of the patients admitted to MICU and SICU over a period of 6 months revealed male preponderance. In the Indian set-up, it is noticed that female population were unwilling to make use of health-care services even if they are critically ill [17]. The average LOS (8 days) and the mean total number of drugs prescribed (10 Nos) for critically ill patients were comparable in both ICUs. The average numbers of AMAs per patient in both ICUs were 3. In our examination in both ICUs, mostly medical patients were admitted in MICU and more surgical and traumatic patients were admitted in SICU.

In both ICUs, 15 AMA monotherapies and nine AMA combination therapies were prescribed to the patients. The extensively prescribed monotherapy in both ICUs was ceftriaxone. The probable reason could be due to its extreme long half-life $(8 \mathrm{~h})$. Piperacillin + tazobactam combination was mostly prescribed AMA in critically ill patients. With respect to the type of drug dosage form, injections were mostly prescribed in both the ICUs.

It was noticed extensive polypharmacy $(100 \%)$ that is more than five drugs were prescribed in all the patients. Polypharmacy is defined as the concomitant use of five or more drugs, and it could enhance drug interactions and drug-related problems [18]. It is essential to keep a balance between the number of drugs and effective pharmacotherapy. More than one AMA were prescribed among (69\%) of the prescriptions. Such inappropriate use of AMAs can result in increased antibiotic resistance. Therefore, health system policymakers should consider implementing reasonable administration guidelines for AMAs through appropriate drug policies [19]. Antimicrobial protocol and guidelines; formulary-based antimicrobial restriction [20] can be used to improve rational usage of antimicrobials. In future, pharmacists have a greater responsibility to take prominent roles in antimicrobial stewardship and infection prevention and control programs in health-care systems.

\section{CONCLUSION}

A comparative study on drug use AMA in MICU and SICU was conducted in tertiary level hospital in South India. The study reveals that a wide
Table 2: AMAs frequently prescribed in the study population

\begin{tabular}{llll}
\hline \multirow{2}{*}{ Drug } & \multirow{2}{*}{ Route } & \multicolumn{2}{l}{ Prescription $\mathbf{n = 5 0}$ (\%) } \\
\cline { 3 - 4 } & & MICU & SICU \\
\hline Ceftriaxone & $\mathrm{P}$ & $13(26.00)$ & $11(22.00)$ \\
Levofloxacin & $\mathrm{O}$ & $3(6.00)$ & $1(2.00)$ \\
Meropenem & $\mathrm{P}$ & $11(22.00)$ & $9(18.00)$ \\
Azithromycin & $\mathrm{P}$ & $1(2.00)$ & $3(6.00)$ \\
Ciprofloxacin & $\mathrm{P}$ & $2(4.00)$ & $2(4.00)$ \\
& $\mathrm{O}$ & $1(2.00)$ & $7(14.00)$ \\
Colistin & $\mathrm{P}$ & $3(6.00)$ & $4(8.00)$ \\
Cefotaxime & $\mathrm{P}$ & $4(8.00)$ & $1(2.00)$ \\
Amikacin & $\mathrm{P}$ & $1(2.00)$ & $1(2.00)$ \\
Clindamycin & $\mathrm{P}$ & $7(14.00)$ & $3(6.00)$ \\
Clarithromycin & $\mathrm{P}$ & $1(2.00)$ & $2(4.00)$ \\
Erythromycin & $\mathrm{O}$ & $1(2.00)$ & $3(6.00)$ \\
Linezolid & $\mathrm{P}$ & $2(4.00)$ & $3(6.00)$ \\
& & $25( \pm 3.859)$ & $25( \pm 3.059)$ \\
\hline
\end{tabular}

P*: Parenteral, $0^{*}$ : Oral. MICU: Medical intensive care unit, SICU: Surgical intensive care unit, SD: Standard deviation, AMA: Antimicrobial agents

Table 3: AMAs prescribed in combination in the study population

\begin{tabular}{llll}
\hline Drug combination & Route & \multicolumn{2}{l}{ Prescription n (\%) } \\
\cline { 3 - 4 } & & MICU & SICU \\
\hline Piperacillin+tazobactam & $\mathrm{P}$ & $11(27.50)$ & $11(28.95)$ \\
Amoxicillin+clavulanic acid & $\mathrm{P}$ & $13(32.50)$ & $14(36.84)$ \\
Meropenem+sulbactam & $\mathrm{P}$ & $9(22.50)$ & $6(15.79)$ \\
Cefoperazone+tazobactam & $\mathrm{O}$ & $5(12.50)$ & $4(10.53)$ \\
Imipenam+cilastatin & $\mathrm{P}$ & $2(5.00)$ & $3(7.89)$ \\
& & $8 \pm 4.47$ & $7.6 \pm 4.472$ \\
\hline
\end{tabular}

MICU: Medical intensive care unit, SICU: Surgical intensive care unit,

SD: Standard deviation, AMA: Antimicrobial agents, $\mathrm{P}^{*}$ : Parenteral, $\mathrm{O}^{*}$ : Oral

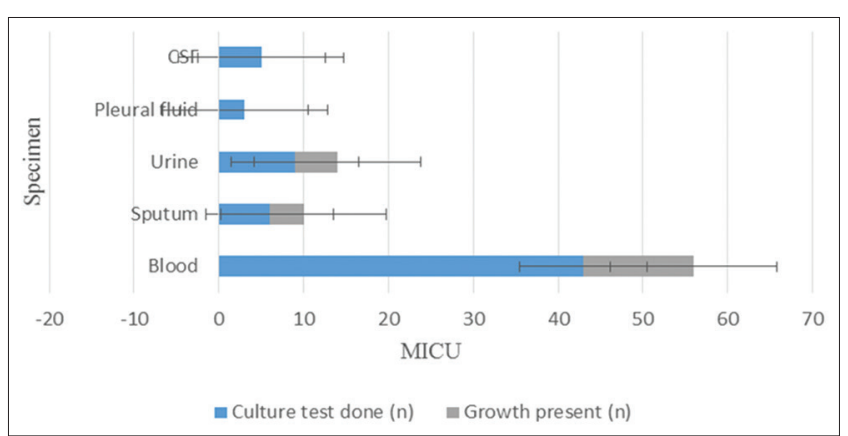

Fig. 3: Pattern of culture in medical intensive care unit

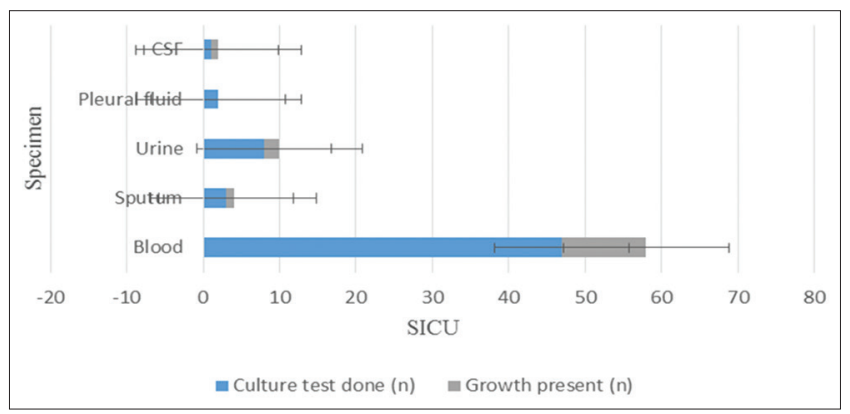

Fig. 4: Pattern of culture in surgical intensive care unit

class and percentage of AMAs were prescribed in ICUs. Majority of patients received more than two AMAs during the ICU stay. As the patients were severely ill, the majority of the AMAs were prescribed 
as injection. Meropenem was the most frequently prescribed monotherapy and amoxicillin + clavulanic acid was the combinative drug used in critically ill patients. The study reveals that appropriate use of AMAs and culture sensitivity. When the doctors prescribed the medicines empirically, they adjusted and deescalated them as soon as feasible. Nevertheless, some antibiotics, especially meropenem, were overused. Clinical pharmacist and physicians must play a crucial role in minimizing the problems associated with irrational use of AMAs by conducting awareness programs among other health-care professionals. The development and regular policies could be useful in promoting better patient care.

\section{ACKNOWLEDGMENT}

The authors would like to thank school of pharmaceutical sciences, Vels University, for granting us necessary permission for the study. The authors also thank all the health-care professionals of ICUs, of for their constant support and guidance.

\section{AUTHORS CONTRIBUTIONS}

Authors make substantial contributions to conception and design, and/or acquisition of data, and/or analysis and interpretation of data; Authors participate in drafting the article or revising it critically for important intellectual content, and Authors give final approval of the version to be submitted and any revised version. N.P. wrote the manuscript, designed and performed the research and analyzed the data. V.S.K. contributed equally to this work and supervisor the project. All authors reviewed the manuscript.

\section{CONFLICTS OF INTERESTS}

The Authors declare no conflict of interests.

\section{REFERENCES}

1. Benjamin B, Kumar BS, Udayakumar P, Swamy VB. Comparative drug utilization of antimicrobial agents in medical and respiratory intensive care units of a tertiary care teaching hospital in South India. Indian J Pharm Pract 2016;2(1):123.

2. WHO. Introduction to Drug Utilization Research. WHO; 2013. Available from: http://apps.who.int/medicinedocs/en/d/Js4876e/.

3. Shanker J, Ghorpode A, Upasani CB. Triage for surgical ICU: Anesthesiologist and intensivist as gatekeepers to ICU. Indian J Crit Care Med 2006;10:167-70.

4. Alamchandani RR, Khubchandani PR. A study evaluating appropriateness of utilization pattern of antimicrobials in patients admitted in medical intensive care unit of tertiary care teaching rural hospital. Int J Pharm Pharm Sci 2015;7:101-104.

5. Luyt CE, Bréchot N, Trouillet JL, Chastre J. Antibiotic stewardship in the intensive care unit. Crit Care 2014;18:480.

6. Khan KA. Drug utilization study of antihyperglycemic agents in outpatients at opd clinics in south indian metropolitan city. Int J Pharm Pharm Sci 2016;8:58-60.

7. Kollef MH. Optimizing antibiotic therapy in the intensive care unit setting. Crit Care 2001;5:189-95.

8. John LJ, Devi P, John J, Guido S. Drug utilization study of antimicrobial agents in medical intensive care unit of a tertiary care hospital. Asian J Pharm Clin Res 2011;4:81-4.

9. Nathiya D, Pandey K, Sharma RK. A study on drug utilization pattern of antimicrobials in outpatient department of medicine at tertiary care hospital. Int J Res Pharm Sci 2014;4:40-5.

10. Shankar PR, Partha P, Shenoy N, Brahmadathan KN. Investigation of antimicrobial use pattern in the intensive treatment unit of a teaching hospital in western Nepal. Am J Infect Control 2003;31:410-4.

11. Biswal S, Mishra P, Malhotra S, Puri GD, Pandhi P. Drug utilization pattern in the intensive care unit of a tertiary care hospital. J Clin Pharmacol 2006;46:945-51.

12. de Rooij SE, Abu-Hanna A, Levi M, de Jonge E. Factors that predict outcome of intensive care treatment in very elderly patients: A review. Crit Care 2005;9:R307-14.

13. Smythe MA, Melendy S, Jahns B, Dmuchowski C. An exploratory analysis of medication utilization in a medical intensive care unit. Crit Care Med 1993;21:1319-23.

14. Shankar PR, Partha P, Dubey AK, Mishra P, Deshpande VY. Intensive care unit drug utilization in a teaching hospital in Nepal. Kathmandu Univ Med J (KUMJ)2005;3:130-7.

15. Hartmann B, Junger A, Brammen D, Röhrig R, Klasen J, Quinzio L, et al. Review of antibiotic drug use in a surgical ICU: Management with a patient data management system for additional outcome analysis in patients staying more than 24 hours. Clin Ther 2004;26:915-24.

16. Usluer G, Ozgunes I, Leblebicioglu H, Turkish Antibiotic Utilization Study Group. A multicenter point-prevalence study: Antimicrobial prescription frequencies in hospitalized patients in Turkey. Ann Clin Microbiol Antimicrob 2005;4:16.

17. Ministry of Home Affairs. Directorate of Census Operations Karnataka Census; 2011. Available from: http://www.censuskarnataka.gov.in.

18. Viktil KK, Blix HS, Moger TA, Reikvam A. Polypharmacy as commonly defined is an indicator of limited value in the assessment of drug-related problems. Br J Clin Pharmacol 2007;63:187-95.

19. ASHP statement on the pharmacist's role in antimicrobial stewardship and infection prevention and control. Am J Health Syst Pharm 2010;67:575-7.

20. Fish DN, Ohlinger MJ. Antimicrobial resistance: Factors and outcomes. Crit Care Clin 2006;22:291-311.

21. Singh N, Yu VL. Rational empiric antibiotic prescription in the ICU. Chest 2000;117:1496-9. 